\title{
Mediastinal ectopic parathyroid adenomas: diagnostic and therapeutic challenge
}

\author{
Massine El Hammoumi, Marius Kamdem, Mouad Amraoui, Mohamed Bhairis, el Hassane Kabiri \\ Department of Thoracic Surgery, Military Teaching Hospital Mohammed V, Rabat, Morocco
}

Kardiochirurgia i Torakochirurgia Polska 2021; 18 (4): 227-230

\begin{abstract}
Introduction: Mediastinal parathyroid adenomas are a rare condition.

Aim: Analysis of epidemiological data, clinical manifestation of mediastinal parathyroid adenoma (MPA), including imaging modalities, and therapeutic approach to this condition.

Material and methods: A retrospective study of documented cases of mediastinal parathyroid adenoma that were managed at our Department of Thoracic Surgery of the Military Teaching Hospital Mohammed V, between January 2010 and December 2019. Results: During a 9-year period in our department, 21 documented cases of MPA were treated surgically. Patients ranged in age from 20 to 69 years, with a mean age of 45 years, and there was no gender predominance. The most frequently reported manifestations were osteoarticular, with bone pain in 66.6\% (14/21), pathological fractures in $28.5 \%$ (6/21), and osteoporosis in $23.8 \%$ (5/21). Cervical ultrasound, MIBI scintigraphy and cervico-thoracic computed tomography scan were performed respectively in 28.5\% $(n=06 / 21)$, in $47.6 \%(n=10 / 21)$ and $100 \%(n=21)$, and allowed positive diagnosis of MPA. All patients benefited from resection surgery, through a classical transverse cervicotomy which was extended in some cases to the manubrium, and in only one case treated with thoracotomy.

Conclusions: Management of mediastinal parathyroid adenoma is based on the diagnosis imaging assessment step, usually by MIBI scintigraphy. Surgical ablation is a radical treatment. Peroperative hormonal evaluation is mainly recommended.
\end{abstract}

Key words: adenoma, ectopic parathyroid, calcemia, surgery.

\section{Introduction}

Primary hyperparathyroidism (PHP) is a common endocrine disease, caused by long-term autonomous and inappropriate excessive production of parathyroid hormone (PTH), by one or more hyperfunctioning parathyroid glands, with the main metabolic consequence of hypercalcemia. It is related in 10-20\% of cases to hyperplasia of glands, in $1 \%$ to a parathyroid carcinoma and in $80 \%$ of cases to a parathyroid adenoma [1]. In 15-20\% of cases, the latter is ectopic, due to an aberrant embryological migration [2]. The most frequent ectopic locations of parathyroid glands are intra-thyroid, para-tracheal and retroesophageal. The mediastinal site is less frequent and accounts for 10-15\% of ectopic parathyroid adenomas [3]. A mediastinal parathyroid gland is defined as a parathyroid lying completely below the level of the clavicle.
Aim
Our study reports the management of 21 cases of medi- astinal parathyroid adenoma (MPA). The objectives of this study were to determine the clinical manifestation of MPA, to analyze the imaging methods performed, and finally to describe the therapeutic approach for our patients.

\section{Material and methods}

This is a descriptive and retrospective study, based on documented cases of MPA, during the period from January 2010 to December 2019 at the Thoracic Surgery Department of the Mohammed V Military Training Hospital in Rabat. The patients included in our study had pathological confirmation of MPA after surgery.

\section{Results}

Patients ranged in age from 20 to 69 years, with a mean age of 45 years. There was no gender predominance. The medical history was dominated by arterial hypertension in $23.8 \%(n=5 / 21)$, renal lithiasis in $14.28 \%(n=3 / 21)$, thyroidectomy in $9.5 \%(n=2 / 21)$, diabetes in $14.2 \%(n=3 / 21)$, cardiac disease in $9.5 \%(n=2 / 21)$, and end-stage renal disease in $4.2 \%(n=1 / 21)$; it is also noted that 6 patients did not have any notable history. The frequently reported manifestations were mainly osteoarticular with bone pain in $52.3 \%(n=11 / 21)$, multiple fractures in $28.5 \%(n=6 / 21)$ and osteoporosis in $14.2 \%(n=3 / 21)$. Preoperative parathyroid hormone (PTH) and blood calcium values of our patients revealed significantly elevated $\mathrm{PTH}$ and hypercalcemia with a mean of $541 \mathrm{pg} / \mathrm{ml}$ and $133.1 \mathrm{mg} / \mathrm{l}$, respectively. 

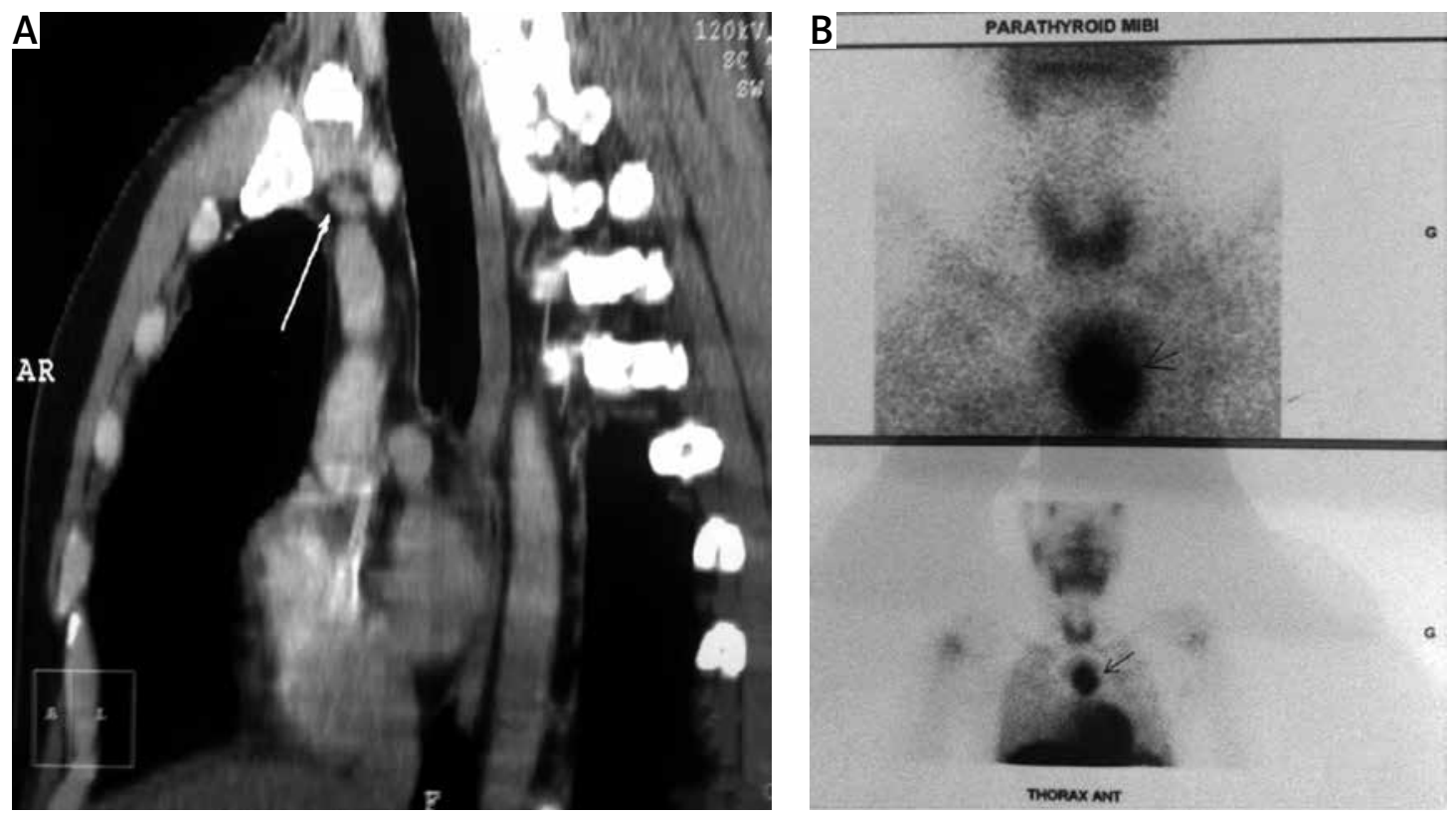

Figure 1. A - Sagittal view of thoracic CT scan shows an upper mediastinal mass neighboring a vascular structure. B - A $99 \mathrm{mT}$ T-MIBI scintigraphy reveals ectopic parathyroid adenoma in the superior mediastinum

Imaging examinations were first cervical ultrasound in $28.5 \%(n=6 / 21)$ giving a presumptive diagnosis of MPA, but in addition it allowed the diagnosis of 2 cases of multi-

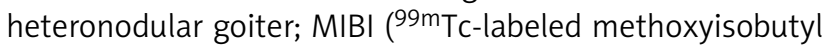
isonitrile) scintigraphy was used in $47.6 \%(n=10 / 21)$ and cervico-thoracic CT scan was used in $100 \%(n=21)$, both offering a positive diagnosis of MPA in 100\% (Figure 1 ).

Six of our patients presented with postoperative hypocalcemia treated with intravenous calcium and then per os intake.

\section{Surgical technique}

Adenoma resection was performed in all cases, associated in two cases with subtotal thyroidectomy. The surgical approach was (Figure 2) a cervicotomy in $19 \%$ of cases $(n=4 / 21)$, a cervico-manubriotomy in $47.6 \%(n=10 / 21)$, subtotal cervico-sternotomy in $9.5 \%(n=2 / 21)$, manubriotomy in $9.5 \%(n=2 / 21)$, sternotomy in $9.5 \%(n=2 / 21)$, thoracotomy in $4.7 \%(n=1 / 21)$.

Whatever the surgical approach, per-operative PTH dosage was done 15 minutes after removal of the pathological gland in the homolateral internal jugular vein. Post-operatively PTH dosage was at $\mathrm{H}_{4}$ and $\mathrm{H}_{24}$. In one patient the PTH level was still high after resection of MPA to become lower after one hour of the post-operative course. We noted a significant drop in value compared to the initial value (Figure 3), with an average value of $47 \mathrm{ng} / \mathrm{l}$ for post-operative dosage at $\mathrm{H}_{24}$. Six of our patients presented severe hypocalcemia post-operatively that was treated with intravenous calcium then per os intake. About $72 \%$ of patients $(n=15)$ were discharged on the third day, but 6 of them left the hospital 7 days after surgery because of persistent hypocalcemia and hungry bone syndrome, a severe but rare form of postoperative hypocalcemia. The one-month, 3-month, 1-year and 2-year follow-up was uneventful.

\section{Discussion}

Mediastinal parathyroid adenomas are rare without gender predominance, as evidenced by our study in agreement with other studies [4]. The classic presentation of parathyroid adenoma is that of hyperparathyroidism associated with psychiatric symptoms, kidney, bone and gastrointestinal stones [4]. However, this complete constellation of symptoms is rarely observed today due to frequent assessments of patients' blood chemistry.

On physical examination, the majority of cervical parathyroid adenomas did not have a palpable mass in the neck. The small size of these adenomas is one of the reasons why a clinician may suspect another disease before looking at the laboratory results. It is the hypercalcemia with concomitant high levels of PTH that confirms the diagnosis of primary hyperparathyroidism. But other biological data should be collected, such as phosphorus levels, vitamin D and others.

Accurate preoperative identification of hyperfunctional parathyroid glands is imperative for satisfactory results, but not all imaging modalities should be used to diagnose this condition. In our study, preoperative diagnosis was obtained by MIBI-Tc ${ }^{99 m}$ scintigraphy, cervico-thoracic CT and cervical ultrasound. The latter is limited by the operator's experience and inability to explore the mediastinum. Moreover, in patients who have never undergone cervicotomy, its sensitivity to detect parathyroid adenoma reaches $80 \%$, but after a first cervical surgery (as was the case in two of our patients) or associated thyroid disease (goiter) sensitivity is reduced to only $30 \%$ [5].

$\mathrm{MIBI}$ scintigraphy is based on the difference in clearance of the $T c^{99 m}-M I B I$ tracer in the thyroid and parathyroid glands. After injection, the tracer is captured early and intensely on both glands, the elimination of the thyroid is faster while the fixation of the parathyroid persists over 

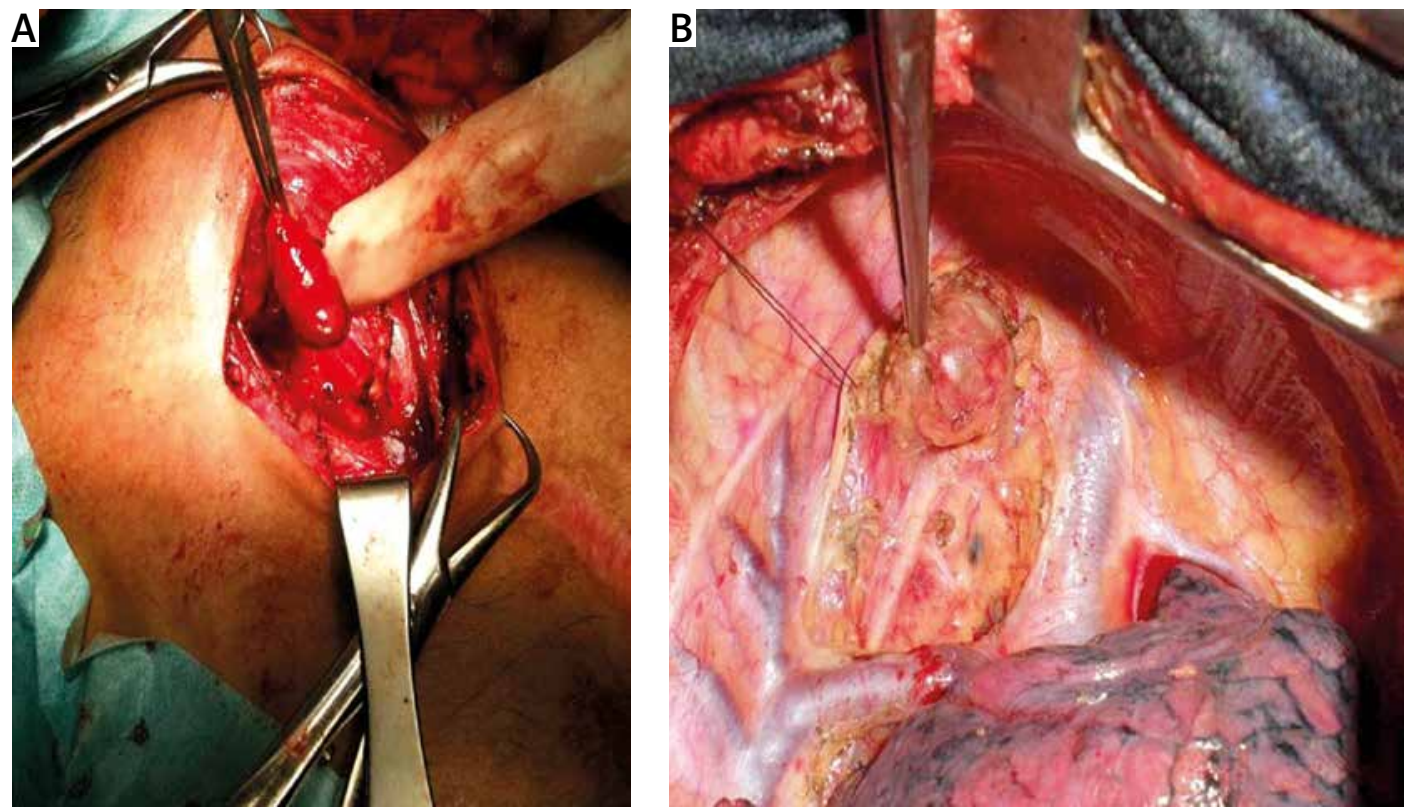

Figure 2. A - Peroperative view of pathological parathyroid adenoma resected via cervicotomy. B - Preoperative view of thoracotomy showing a superior inter-tracheoesophageal parathyroid adenoma

time. Therefore, MIBI scintigraphy is considered positive because of the existence of a focus of hyperfixation at an early stage, the intensity of which persists or even increases with time. We also noted that some authors have shown that the sensitivity of MIBI scintigraphy improves when the weight of the gland is greater than $600 \mathrm{mg}$ [6], allowing cervical and mediastinal exploration in search of parathyroid pathology.

Despite the fact that MRI provides good contrast for soft tissue study, cervicothoracic CT with contrast has been used to accurately localize ectopic parathyroid adenoma, with an estimated sensitivity of 75\% [7]. Recently, fluorocholine (FCH) PET/CT offers a good tool in parathyroid gland exploration. Behera and Damle explained the increasing role of FCH PET/CT over MIBI scintigraphy because of the better spatial resolution and the detection of smaller adenomas [8]. Regarding location, upper parathyroid gland ectopias are less frequent (1\%), and can be found in the retropharyngeal spaces, compared with lower parathyroid gland ectopias that can be found throughout the large descending area with the thymus, from the hyoid bone at the top to the pericardium at the bottom, from the prevertebral plane at the back to the plane of the infrahyoid muscle and sternum at the front [9].

Mediastinal ectopic parathyroid adenomas are a recognized cause of persistent hyperparathyroidism, and hypercalcemia in approximately $0.8 \%$ of iterative parathyroidectomies.

There is no curative medical treatment; the treatment of choice is surgery to resect the pathological glands. This removal surgery can become delicate depending on site and surrounding structures. In addition, there are questions about the benefits of surgery in asymptomatic patients; in response, the Fourth International guidelines for the management of asymptomatic adenomas have published indi-

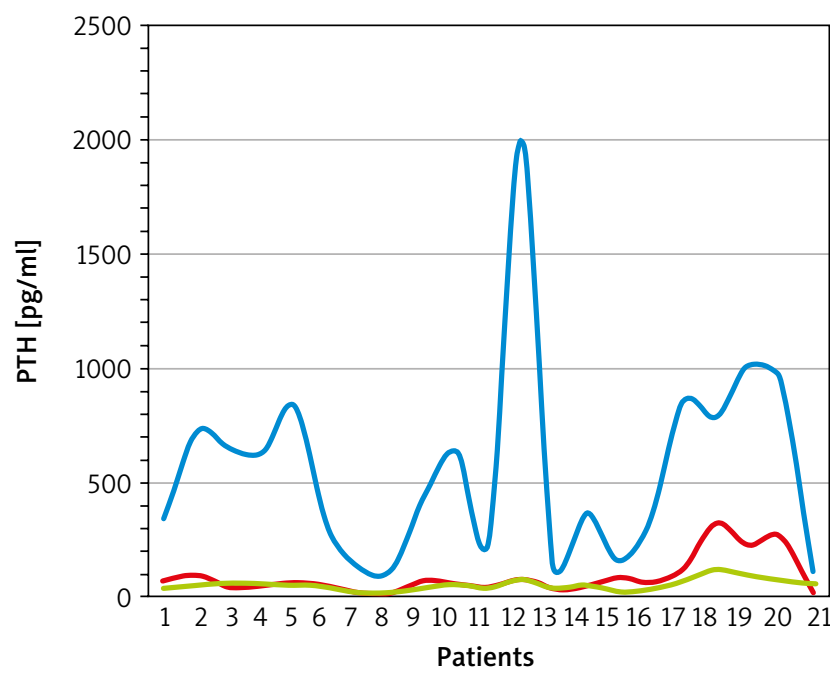

- PTH preop. $\quad$ PTH postop. $\mathrm{H} 4-$ PTH postop. $\mathrm{H} 24$

Figure 3. Kinetics of values of PTH dosage

cations for surgery in asymptomatic patients [10]. Once the indication is established, several types of surgical procedures can be used depending on multiple factors. In our setting, conventional surgery for the management of MPA is still widely used, but recently new approaches are being developed to minimize interventions and reduce complications and repeated surgery. Elsewhere it includes minimally invasive parathyroidectomy, which includes directed parathyroidectomy, endoscopic parathyroidectomy (endoscopic, video-assisted and robotic total parathyroidectomy) and isotope-guided parathyroidectomy. Most of these surgical approaches are recommended for solitary lesions. However, while preoperative imaging may be negative in approximately $30 \%$ of cases [7], the risk in some settings of encountering multi-glandular lesions may necessitate 
exploration by conventional surgery. A large proportion of mediastinal parathyroid adenomas can be successfully removed through a cervical approach. However, if this approach is not possible, a total or partial sternotomy is required and is associated with increased postoperative pain, prolonged hospital stay and complications in up to $21 \%$ of patients.

When a tracer is not used for surgery (radio-guided parathyroidectomy), intraoperative location of the parathyroid gland requires the surgeon's experience to discriminate a normal parathyroid gland and a pathological, hypervascularized, darker parathyroid gland [10-15], both orthotopic or ectopic. Once the pathological parathyroid gland has been identified, it is extracted with great care; it is necessary to completely remove an intact gland by slowly grasping its capsule until the vascular pedicle is identified and then ligated $[16,17]$.

Once excision has been performed, regardless of the approach, the intraoperative PTH dosage and its kinetics are essential to ensure complete excision of the pathological parathyroid gland. Postoperatively, large MPAs may be associated with a higher incidence of postoperative hypocalcemia [18-21] due to hungry bone syndrome (six in our study). Patients with small MPAs were less likely to develop postoperative hypocalcemia.

\section{Conclusions}

Parathyroid adenoma can occur in a mediastinal ectopic situation. It therefore represents a particular challenge at both the diagnostic and therapeutic levels. The management is based on imaging assessment, which is an essential step, since it determines the precise location and number of hyper-functioning parathyroid glands, providing exact cervico-mediastinal mapping, which will allow the surgeon to perform a targeted "surgical strike", by adapted ways.

\section{Disclosure}

The authors report no conflict of interest.

\section{References}

1. Babey M, Kopp P. Primary hyperparathyroidism. Forum Med Suisse 2009; 9 : 791-797.

2. El Guich D, Jaidane A, Riahi M, Zouaouic, Ouertani H. Adénome parathyroïdien médiastinal. Annales d'Endocrinologie 2018;79: 414-434.
3. Cakmak H, Tokat AO, Karasu S, Özkan M. Giant mediastinal parathyroid adenoma. Tuberk Toraks 2011; 59: 263-265.

4. Hariga I, Zribi S, Khamassi K, Ben Gamra O, Skouri Y, Ben Amor M, Mbarek C, El Khedim A. Prise en charge de l'hyperparathyroïdie primaire: à propos de 25 cas et revue de la littérature. J Tun Orl 2007; 12: 19.

5. Bienvenu M, Amar L, Vignaux O. Diagnostic des adénomes parathyroïdiens ectopiques médiastinaux: Apport de l'IRM cardiaque. J Radiol 2003; 84: 19691973.

6. Acín-Gándara D, Pereira-Pérez F, Medina-García M, Sebastián-Viana T. Factors infuencing the sensitivity of ultrasound and gamma location of the parathyroid adenoma. Cir Esp 2020; 98: 18-25.

7. Policeni BA, Smoker WRK, Reede DL. Anatomy and embryology of the thyroid and parathyroid glands. Semin Ultrasound CT MR 2012; 33: 104-114.

8. Behera A, Damle NA. Incremental role of $18 \mathrm{~F}$-fluorocholine PET/CT over technetium-99m-labeled MIBI scan in hyperparathyroidism. Indian J Endocrinol Metab 2016; 20: 888-890.

9. Eugenio P, Johan F. Parathyroidectomie. The Open Access Atlas of Otolaryngology, Head \& Neck Operative Surgery. 2017.

10. Claire Blanchard-Louis. Les symptômes non spécifiques et qualité de vie dans l'hyperparathyroïdie primaire modérée: étude prospective, multicentrique, non randomisée, avant et après chirurgie: résultats à un an. Thése de médecine 2012 p 36.

11. Bilezikian JP, Brandi ML, Eastellet R, Silverberg SJ, Udelsman R, Marcocci C, Potts Jr JT. Guidelines for management of asymptomatic primary hyperparathyroidism: summary statement from the Fourth International Workshop. J Clin Endocrinol Metab 2014; 99: 3561-3569.

12. Spanheimer PM, Stoltze AJ, Howe JR, Sugg SL, Lal G, Weigel RJ. Do giant parathyroid adenomas represent a distinct clinical entity? Surgery 2013; 154: 714-718.

13. Krishnamurthy A, Raghunandan GC, Ramshankar V. A rare case of giant parathyroid adenoma presenting with recurrent episodes of pancreatitis. Indian J Nucl Med 2016; 31: 36-38.

14. Sisodiya R, Kumar S, Palankar N. Case report on giant parathyroid adenoma with review of literature. Indian I Surg 2011; 75: 21-22.

15. Asghar A, Ikram M, Islam N. A case report: giant cystic parathyroid adenoma presenting with parathyroid crisis after Vitamin D replacement. BMC Endocr Disord 2012; 12: 14.

16. Sahsamanis G, Gkouzis K, Samaras S, Pinialidis D, Dimitrakopoulos G. Surgical management of a giant parathyroid adenoma through minimal invasive parathyroidectomy. A case report. Int J Surg Case Rep 2017; 31: 262-265.

17. Salehian M, Namdari O, Mohammadi SS, Feazli YH. Primary hyperparathyroidism due to a giant parathyroid adenoma: a case report. Int J Endocrinol Metabol 2009; 9: 101-105.

18. Castro MA, López AA, Fragueiro LM, García NP. Giant parathyroid adenoma: differential aspects compared to parathyroid carcinoma. Endocrinol Diabetes Metab Case Rep 2017; 2017: 17-0041.

19. Haldar A, Thapar A, Khan S, Jenkins S. Day-case minimally invasive excision of a giant mediastinal parathyroid adenoma. Ann R Coll Surg Engl 2014; 96 : 21-23.

20. Mantzoros I, Kyriakidou D, Galanos-Demiris K, Chatzakis C, Parpoudi SN, Sapidis N, Loutzidou L, Ioannidis O, Angelopoulos S, Tsalis K. A rare case of primary hyperparathyroidism caused by a giant solitary parathyroid adenoma. Am J Case Rep 2018; 19: 1334-1337.

21. Taghavi Kojidi H, Vagharimehr N, Mohseni S, Pajouhi M, Mohajeri-Tehrani MR. Unusual ectopic parathyroid adenoma: a case report. Acta Med Iran 2016; 54: 547-550. 